\title{
IMAGE MINING: CONCEITOS E TÉCNICAS
}

\author{
Diogo Floriano \\ diiogofloriano@gmail.com
}

\section{Resumo}

A facilidade em armazenar imagens e a grande concentração de imagens em bases de dados impulsionou a geração de aplicações que envolvessem a consulta, recuperação e análise dessas imagens. Para se extrair informações de uma base de imagens são necessários algoritmos que possam ser aplicados a uma grande massa de dados, que ao mesmo tempo classifique e encontre padrões em relacionamentos. Para que a mineração de imagens, como também dados em multimídia se torne possível existe uma interdisciplinaridade que liga esse conceito, e tudo será abordado neste artigo.

Palavras-chave:Data mining. Image mining. KDD.

\section{Introdução}

A grande concentração de dados em multimídia disponíveis no momento está se tornando cada vez mais acesível, e tendo a internet como sua catalisadora, o torna ainda mais expressivo nos dias de hoje. Além de outros avanços científicos como pesquisas que envolvem visão computacional, medicina ou sensoriamento remoto, envolvem a mineração de imagens. A partir disto diversas tecnologias evoluiram para suprir as necessidades desta área para que seja possível extrair dados uteis e resumidos destas informações (VIEIRA, 2002).

Para que uma imagem seja armazenada, ela precisa passar por processos de segmentação e representação. E o resultado deste pré-processamento é uma enorme quantidade de imagens que precisam de alguma forma serem consultadas, recuperadas ou analisadas, que onde entra a mineração de imagens. Também existem outras técnicas que podem ser aplicadascomo o SRIC (Sistema de Recuperação de Imagens por Conteúdo) e a visão computacional, que possui foco na interpretação de imagens com o objetivo de reconhecer objetos ou cenário (VIEIRA, 2012).

Este artigo está estruturado em duas partes, no inicio há uma introdução a mineração de dados em imagens que é o foco deste trabalho, seguido por um detalhamento entre as disciplinas que envolvem essa mineração, e por fim um conclusão sobre o assunto e o que foi possível abstrair deste trabalho.

\section{Conceitos}

Para que o processo de reconhecimento de imagens seja eficiente é necessário que as características que foram extraídas não sejam modificadas, como tamanho, rotação e translação. Esse processo pode ser visto na figura 1, que representa as etapas que são necessárias. Após a seleção das imagens feita em uma base de dados, vem o pré-processamento que aumenta a qualidade dos dados, submetendo a imagem a diversos transformações para extração de características importantes referentes a imagem. Com as informações obtidas é possível realizar a mineração dos dados com técnicas específicas para se descobrir padrões significativos. Então, esses padrões são avaliados e interpretados para obtenção do conhecimento, que pode ser aplicado na tomada de decisões, entendimento de problemas ou em outras atividades estratégicas (SILVA, CÂMARA, 2003). 


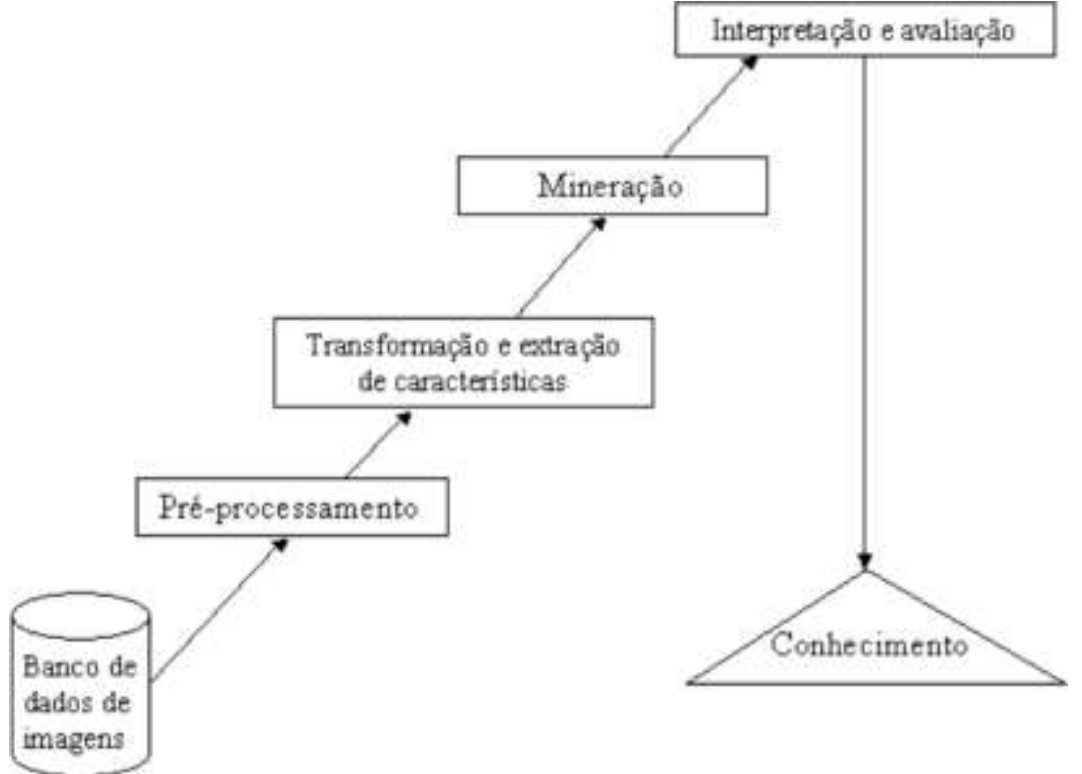

Figura 1 - Processo de mineração de dados em imagens (SILVA, CÂMARA 2003).

Mesmo que a figura 1 se assemelha ao processo de mineração de dados comum, a mineração de dados de imagens não consiste apenas na aplicação de técnicas convencionais para mineração de dados, há diferenças que entre esses dois tipos, entre eles:

- Valores relativos e valores absolutos: em bancos de dados relacionais, os valores que os dados podem assumir são semanticamente significativos. Já valores de imagens não possuem significância sem um suporte de contexto ou formatação.

- Informação espacial: a informação espacial é importante para a interpretação da imagem, diferente dos bancos de dados relacionais.

- Interpretação única e interpretação múltipla: a interpretação múltipla dos padrões visuais é uma característica das imagens. Desta forma, algoritmos tradicionais de associação de padrões não são aplicáveis. O que acarreta em novas categorias de algoritmos, que possa suprir as demandas especiais quando se realiza a mineração e interpretação de padrões a partir de imagens.

- Representação visual dos padrões descobertos: a representação dos padrões de imagens deve ser feita da forma que a informação contextual e espacial seja retida no esquema de representação. As características relevantes da imagem devem ser utilizadas de forma significativa para que o processo de mineração visualmente eficaz (SILVA, CÂMARA, 2003).

Segundo Zhang et al, o objetivo da mineração de dados em imagem é "determinar como a representação de baixo nível (pixels) de uma imagem pode ser eficientemente e efetivamente processada para identificar objetos e seus relacionamentos". Para que os algorítmos tradicionais de mineração de dados funcionem para a mineração de imagens é necessária adaptar os mesmos nos seguintes fatores:

- Valores relativos e absolutos: um campo numérico em um banco de dados possui valor estático, diferente de uma imagem que depende dos valores que estão próximos, o que gera um significado semântico diferente;

- Informação espacial: bancos de dados relacionais não possuem suporte a este tipo de informação e precisam ser adaptados;

- Múltiplas interpretações: imagens dependem totalmente de um contexto para obter um sentido ou significado;

- Representação de padrões: a representação dos padrões abstrarídos deve tratar do contexto e das informações espaciais; 
- Seleção de características: é importante para a mineração de imagens saber escolher quais são as características mais significativas, e;

- Visualização de padrões: a visualização de padrões convencional não é aplicado a mineração de imagens é preciso criar novas formas de visualização para os padrões encontrados (ZHANG et al, 2001).

A mineração de dados em imagens, como a mineração de dados convencional, necessita passar por várias fases dentro do seus processo, entre elas esta a obtenção dos dados, o préprocessamento, a extração das características e a integração (SILVA, CÂMARA, 2003).

\section{Processo de mineração de imagens}

O processo de mineração de dados em imagens faz parte de um processo maior chamado KDD (Knowledge-Discovery in Databases, traduzido para português, Extração de Conhecimento em Base de Dados), que define o esqueleto do processo de mineração, que possui a obtenção dos dados a serem analisados, descoberta de padrões, sua aplicação e validação.

O KDD define a base de qualquer mineração de dados, e cada especialização nessa mineração, como é no caso de imagens, outras atividades são adicionadas para este processo. $\mathrm{Na}$ Figura 2 é identificado que em casos de imagens, também é adicionado mais atividades ao processo de KDD, como etapas das área de visão computacional, SRIC e processamento de imagens (VIEIRA, 2002).

\subsection{Obtenção}

A obtenção dos dados é a fase em que eles é escolhida a base de dados para realizar a mineração com foco em algum objetivo. No caso de um banco de dados de imagem, essa fase é bem evidente, pois se inicia quando qualquer imagem é adquirida. Outra possibilidade é aproveitar a própria Internet que já possui uma base de imagens livre. Também é possível combinar imagens de outras naturezas e de origens, como imagens geográficas obtidas através de renderização ou qualquer outro processo (VIEIRA, 2002).

\subsection{Pré-processamento}

Pelo fato dos dados que serão minerados serem incompletos, ruidosos ou inconsistentes, conforme ilustra a Figura 2, antes de se realizar a integração, os dados devem passar por processos de limpeza, seleção e transformação (ADRIAANS, ZANTINGE, 1996). A limpeza consiste na eliminação ou filtragem de dados inconsistentes ou com algum ruído. A seleção é a eliminação de atributos redundantes e redução da dimensão dos atributos para torná-los mais simples. Esse processamento ocorre em cada imagem antes da integração, através de técnicas de redução de ruído, suavização e aplicação de filtros (GONZALES, WOODS, 1992).

Da mesma forma em que os dados são filtrados e selecionados, também é necessário adaptálos aos algoritmos de mineração de dados. Pois, não é comum trabalhar diretamente com mineração de pixels. Portanto, como parte do pré-processamento, é importante converter as imagens e as suas características para um esquema de representação de dados, seja uma tabelas, registros, atributos ou estruturas de dados (VIEIRA, 2002). 


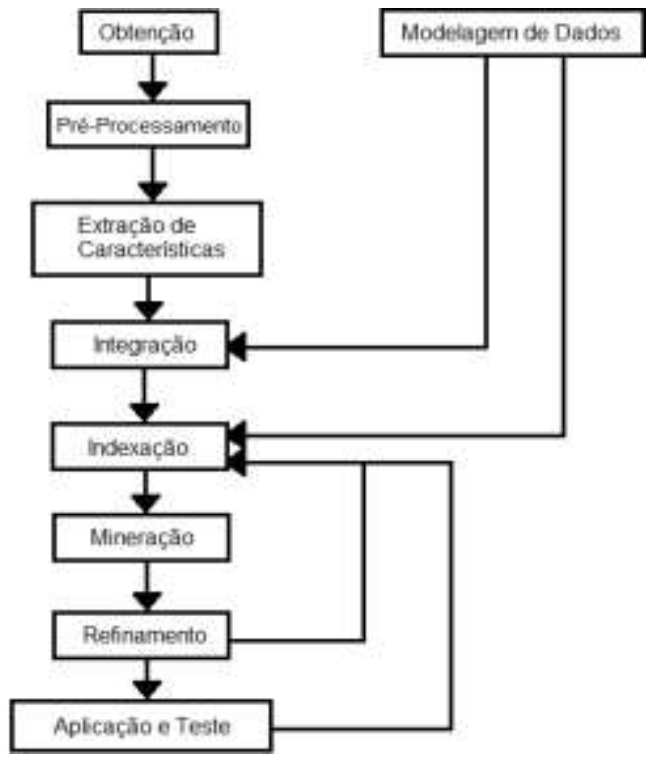

Figura 2 - Processo de Mineração de Imagens(VIEIRA, 2002)

\subsection{Extração de características}

Essa é uma etapa que surgiu exclusivamente para a mineração de imagens. Como não é importante trabalhar diretamente com pixels e sim com uma representação dos dados. Pode ser abordada uma técnica para trabalhar com características de baixo nível como cor, forma e textura, representadas através de vetores de características. Segundo Vailaya, os métodos para extração de características se dividem em dois grupos: os que preservam a informação espacial e aqueles que não a preservam. Existem três abordagens para extração de características que são definidas por Khoshafian e Barei:

a) extração manual, através da entrada de dados feita pelo usuário;

b) extração automática, através de métodos de reconhecimento de padrões, segmentação e outros algoritmos, e;

c) extração híbrida, em que o sistema extrai as informações e o usuário as valida.

Para fazer a análise de cor, as principais técnicas são o histograma, os momentos e as matrizes de co-ocorrência. Ainda segundo Vailaya, a extração de cor descreve as propriedades globais de uma imagem, pois não variam quandofeito alterações no tamanho, porém não contém informações espaciais da imagem. A forma permite obter informações locais e geométricas, intolerantes a variações de escala, orientação e translação. A textura é um conjunto de propriedades de uma imagem, tais como uniformidade, densidade, aspereza, regularidade e intensidade, obtidas por meio da análise da matriz de co-ocorrência, M-SAR e wavelets (VIEIRA, 2002).

\subsection{Integração}

Com as imagens pré-processadas e com as características geradas, é preciso consolidar as informações obtidas pela fase de modelagem. A fase de consolidação é fortemente ligada ao processo de pré-processamento, que envolve a redução de dimensão ou o uso de métodos de transformação para a redução do número de variáveis que representam os dados (FAYYAD et al. 1996).

Como a redução de dimensão, entende-se a escolha dos atributos que melhor representam a imagem, permitindo diminuir a quantidade de dados que passará pelas fases de indexação e 
mineração, por assim reduzir o processamento e quantidade de memória utilizada (VIEIRA, 2002).

\section{Considerações Finais}

O grande crescimento de dados em muiltimídia, devido a popularização da internet e outras áreas que também cresceream, como a visão computacional, a medicina, o georreferenciamento, o sensoriamento remoto e etc, tornam a mineração de dados em imagens cada vez mais importante. O que contribuiu também para a evolução de diversas tecnologias, que são necessárias para suprir as necessidades que a mineração de imagens precisa para que extrair dados uteis e resumidos das informações (VIEIRA, 2002).

A mineração de dados em imagens utiliza de matemática e inteligência artificial, assim como o processo de mineração de dados convencional, para que ele possa organizar, recuperar e analisar os dados e informações que são obtidos através da imagem, tudo isso sem a intervenção do usuário. Além destas disciplinas existe o apoio de sistemas especialistas e outras estruturas de inteligência artificial para representar o que foi aprendido e utilizá-lo para obter novos conhecimentos (SILVA, CÂMARA, 2003).

Para toda e qualquer imagem armazenada, ela passa por processos de segmentação e representação, chamado de pré-processamento. E o resultado disto é uma enorme quantidade de imagens que precisam de alguma forma serem consultadas, recuperadas ou analisadas, que onde entra o processo de mineração de imagens. Também existem outras funcionalidade como o SRIC, que busca a recuperação e consulta de imagens,e a visão computacional, que possui foco na interpretação de imagens com o objetivo de reconhecer objetos ou cenários. (VIEIRA, 2012).

\section{Referências}

ADRIAANS, P.; ZANTINGE, D. Data mining. Syllogic, 1996.

GONZALES, R.C.WOODS, R.E. Processamento de imagens digitais. EdgardBlücher, 1992.

KHOSHAFIAN, S.; BAREI, A.B. Multimedia and image databases.Morgan Kaufmann, 1996.

SILVA, M. P dos Santos, NETO, G. C. Mineração de Dados em Imagens: da arquitetura à ontologia. 2003.

VAILAYA, A. Semantic classification in image databases. Teste de Doutorado, Department of Computer Science, Michigan State University, 2000

VIEIRA, E. V. Mineração de Imagens: conceitos e aplicações em sistemas de recuperação de imagens por conteúdo. Dissertação para Mestrado no Programa de Pós-Graduação em Informática, Setor de Ciências Exatas - Universidade Federal do Paraná, 2002.

ZHANG, J.; HSU, W.; LEE, M. L. Image mining: issues, frameworks and techniques. Proceedings of the 2nd International Workshop on Multimedia Data Mining.EUA, 2001. 